\title{
COMUNICACIÓN
}

\section{Censo de Ornithodoros amblus Chamberlin (Acarina: Argasidae) en la isla Mazorca, Lima, Perú}

\author{
JOSÉ IANNACONE* y LILIANA AYALA* \\ CENSUS OF Ornithodoros amblus CHAMBERLIN (ACARINA: ARGASIDAE) \\ IN MAZORCA ISLAND, LIMA, PERU
}

The aim of the current research was to evaluate the tick Ornithodoros amblus Chamberlin in Mazorca Island, Lima, Peru and its relationship with the presence of seabirds, intensity of guano and wind. The tick samplings were performed between 28 and 29 February of 1998. On each point of sampling was placed during three minutes a black coarse cotton cloth of $0,35 \mathrm{~m}^{2}$. O. amblus showed a spatial clumped distribution. Abundance of ticks was not related with the presence of colonies of birds. Only larvae of $\boldsymbol{O}$. amblus were in relationship with colonies of seabirds. The preference of different growth states of $\boldsymbol{O}$. amblus follow the next sequence: Guanay Cormorant $>$ Peruvian Pelican $>$ Camanay Booby. Different growth states did not found relation with intensities of guano. A preference of tick population of $\boldsymbol{O}$. amblus was observed in relation with strong intensities of winds. The effects of Niño 1997-1998 related to this tick specie is discussed.

Key words: guano seabirds, island, Ornithodoros, survey, tick.

\section{INTRODUCCIÓN}

Las garrapatas forman un grupo de ácaros ggrandes hematófagos pertenecientes principalmente a las familias Argasidae e Ixodidae ${ }^{1,2}$. Dentro de la familia Argasidae "garrapatas de cuerpo blando", el género Ornithodoros es el más numeroso y el más importante como parásito de muchas especies de aves ${ }^{3}$. Los efectos de las altas infestaciones de Ornithodoros sobre diversas especies de aves son variados, tales como deserción de colonias, mortalidad de juveniles y restricción del área de anidación ${ }^{1,4,5}$

En el Perú, Ornithodoros amblus es una especie que parasita varias especies de aves marinas como guanay (Leucogarbo bougainvillii [Lessoný] Phalacrocoracidae), piquero (Sula variegata [Tschudi], Sulidae), el camanay (Sula nebouxii [Lesson], Sulidae), pelicano (Pelecanus thagus [Lesson], Pelecanidae) y zarcillo (Larosterna inca Lesson y Garnot, Laridae) ${ }^{5}$. Se distribuye desde la Isla Lobos de Tierra en Lambayeque $\left(06^{\circ} 28^{\prime} \mathrm{LS}, 80^{\circ} 50^{\prime} \mathrm{LW}\right)$ hasta las Islas Ballestas, en el departamento de Ica $\left(13^{\circ} 44^{\prime}\right.$ LS, $76^{\circ} 24^{\prime} \mathrm{LW}$ ) y ha sido colectada en diecisiete localidades en cinco departamentos del Perú ${ }^{6,7}$. Inclusive ha sido colectado en las costas norte de Chile en los nidos de gaviotas del género Larus spp. ${ }^{8}$.

En una revisión del panorama global referente

\footnotetext{
* Laboratorio de Ecofisiología Animal, Facultad de Ciencias Naturales y Matemáticas, Universidad Nacional Federico Villarreal.

E-mail: joseiannacone@hotmail.com
} 
a esta garrapata parásita de aves guaneras, en la que señalan como perspectiva de futuras investigaciones la cuantificación de sus poblaciones en las islas y puntas guaneras con un adecuado método de muestreo ${ }^{5}$. Se señala como método de muestreo para $O$. amblus, la colecta de cucharones de guano y piedras para el censo de adultos y ninfas de esta garrapata ${ }^{6,9}$. Esta garrapata pasa un periodo de tiempo de su ciclo de vida sumamente corto sobre el ave hospedera $(3,72 \%$ a $6,61 \%)$, mayormente en la etapa larval ${ }^{5}$.

Por otro lado, Se ha realizado una revisión de la importancia de Ornithodoros como vector de enfermedades virales en el ser humano ${ }^{1}$ y su rol en la hematofagia en sus hospederos ${ }^{10}$.

Dentro de las principales Islas Guaneras, de un total de 17, en la Costa Peruana, destacan por su producción de guano y por la cantidad de Aves guaneras, la Isla Mazorca, Huacho, Perú. Sin embargo, en la Costa Central del Perú los invertebrados terrestres en las islas son poco estudiados ${ }^{11}$.

Por lo que el objetivo de la presente investigación fue evaluar la distribución espacial de $O$. amblus en la Isla Mazorca, Lima, Perú y su relación con la presencia de aves marinas, tipo de sustrato e intensidad de viento.

\section{MATERIAL Y MÉTODOS}

El área de estudio comprendió la Isla de Mazorca (11 $\left.{ }^{\circ} 23^{\prime} 30^{\prime \prime} \mathrm{LS}, 77^{\circ} 26^{\prime} 30^{\prime \prime} \mathrm{LW}\right)$, frente a Huacho en el departamento de Lima, Perú. Su extensión es de 11,63 ha. El muestreo de las garrapatas se realizó los días 28 y 29 de febrero del año 1998, entre las 9:00 h y las 12:00 h, debido a que durante estas horas, la abundancia de este ácaro es mayor en el guano o bajo las piedras". La temperatura promedio del agua y del aire fue de $24^{\circ} \mathrm{C}$ y $26,2^{\circ} \mathrm{C}$, respectivamente.

La isla fue dividida en cuatro cuadrantes, tomando como punto de referencia el faro que se encuentra en la parte alta de la isla, se fijó cada uno de los 25 puntos al azar, con estacas colocadas aproximadamente cada $30 \mathrm{~m}$. En cada punto se tomaron los siguientes datos: presencia de colonias de aves y tipo de ave (por observación directa con binoculares), tipo de sustrato en cuatro categorías (guano abundante: aquella que presenta un sustrato oscuro y húmedo. Lugar frecuente de aposentamiento de las aves guaneras; guano medio: Es aquella zona donde la colonia de aves presentan un menor número de individuos, generalmente hacia los alrededores de ésta, guano pobre: Es aquella área donde se aposentan aves en número escaso y no forman parte de las colonias principales; y finalmente ausencia de guano: que no son lugares de aposentamiento, como por ejemplo zonas cercanas a barrancos) e intensidad de viento en tres categorías (fuerte, medio y ausente). Además, en cada punto de muestreo se colocó por espacio de tres minutos una tela de tocuyo negra de $0,35 \mathrm{~m}^{2}$ (modificado por Beaty ${ }^{12}$ ), la que luego fue puesta en un frasco de un litro de capacidad de boca ancha con alcohol etílico al $70 \%$.

El contenido de cada frasco se examinó en el laboratorio de Ecofisiología Animal de la Facultad de Ciencias Naturales y Matemáticas de la Universidad Nacional Federico Villarreal. Para su identificación taxonómica se usó descripciones previas $^{5,13}$. Los especimenes de O. amblus fueron separados por estado de desarrollo en: larvas, ninfas, adultos (machos y hembras).

\section{Análisis de Datos}

Se determinó la abundancia promedio en base al número de especimenes de $O$. amblus de cada estadio por separado o en total sobre el número total de puntos de muestreo censados $(n=25)$. El coeficiente de dispersión (CD) empleado para determinar su distribución espacial, se determinó de la relación entre Varianza $\left(\mathrm{S}^{2}\right)$ /Abundancia media.

Se realizó la prueba de Chi-cuadrado $\left(\mathrm{c}^{2}\right)$ para determinar la relación existente entre la abundancia de garrapatas y la presencia de colonias de aves, tipo de aves, intensidad de sustrato y de viento. Para el cálculo de las pruebas estadísticas descriptivas e inferenciales se usó el paquete estadístico SPSS 10 para Windows 98.

\section{RESULTADOS}

El número total de garrapatas censadas fue de 70 O. amblus y un ejemplar adulto de Ornithodoros spheniscus. La abundancia promedio de garrapatas en cada estado desarrollo presentó el siguiente orden decreciente: ninfal > adulto $>$ larval (Tabla 1). El 72\% $(\mathrm{n}=18)$ del total de muestras censadas $(n=25)$ presentaron O. amblus. El CD fue de 5,10, lo cual nos señala que $O$. amblus presenta una distribución espacial 
amontonada. La Tabla 2 nos indica que la abundancia de garrapatas no estuvo relacionada con la presencia o ausencia de colonias de aves $\left(\chi^{2}=4,76 ;\right.$ g. $\left.1 .=3 ; \mathrm{P}=0,18\right)$. Sin embargo, las larvas de $O$. amblus estuvieron más relacionadas con la presencia de colonias de aves. La Figura 1 muestra el número promedio de garrapatas y su relación con las especies de aves marinas en la Isla Mazorca: Siendo el orden decreciente de preferencia de la siguiente forma: Guanay $>$ Camanay > Pelícano > Piquero. La Tabla 3 nos muestra la preferencia de los diferentes estados de desarrollo de $O$. amblus al Guanay $>$ Pelícano $>$ Camanay $\left(\chi^{2}=23,66 ;\right.$ g. $\left.1 .=9 ; \mathrm{P}=0,0006\right)$. Se observa la ausencia de garrapatas asociada al Piquero. Los diferentes estados de desarrollo de no se encontraron en relación con las categorías de guano $\left(\chi^{2}=7,28 ;\right.$ g. $\left.1 .=9 ; \mathrm{P}=0,60\right)$. Sin

Tabla 1. Abundancia de larvas, ninfas y adultos de la garrapata Ornithodoros. amblus

\begin{tabular}{lcc}
\hline $\begin{array}{l}\text { Estado de } \\
\text { desarrollo }\end{array}$ & $\begin{array}{c}\text { Abundancia promedio* } \pm \text { DS } \\
\text { (Número de muestras, Porcentaje } \\
\text { de muestras con garrapatas) }\end{array}$ & $\begin{array}{c}\text { Abundancia total } \\
\text { (Abundancia porcentual (\%)) }\end{array}$ \\
\hline Larva & $0,5 \pm 1,1(05,20 \%)$ & $12(17,14)$ \\
Ninfa & $1,4 \pm 2,3(12,48 \%)$ & $34(48,57)$ \\
Adulto total & $1 \pm(07,28 \%)$ & $24(34,29)$ \\
Hembra & $0,7 \pm 0,9(07,28 \%)$ & $13(19,06)$ \\
Macho & $0,4 \pm 1,1(04,16 \%)$ & $11(09,94)$ \\
\hline
\end{tabular}

* = determinado en base al número total de puntos de muestreo $(\mathrm{n}=25)$.

DS = Desviación estándar.

Tabla 2. Abundancia de la garrapata Ornithodoros amblus por estados de desarrollo asociada a las colonias de aves

\begin{tabular}{|c|c|c|c|c|c|}
\hline $\begin{array}{l}\text { Colonias } \\
\text { de aves }\end{array}$ & Larva & Ninfa & Hembra & Macho & Total \\
\hline Ausencia & $3(25 \%)$ & $15(44,12 \%)$ & $8(61,53 \%)$ & $7(63,64 \%)$ & $33(46,67 \%)$ \\
\hline Presencia & $9(75 \%)$ & $19(55,88 \%)$ & $5(38,47 \%)$ & $4(36,36 \%)$ & $37(53,33 \%)$ \\
\hline
\end{tabular}

Tabla 3. Abundancia y valores porcentuales de la garrapata Ornithodoros amblus asociada a cuatro diferentes especies de aves marinas

\begin{tabular}{|c|c|c|c|c|c|}
\hline Aves marinas & Larva & Ninfa & Hembra & Macho & Total \\
\hline Guanay & $3(33,33 \%)$ & $17(89,48 \%)$ & $4(80 \%)$ & $4(100 \%)$ & $28(75,67 \%)$ \\
\hline Camanay & $0(0 \%)$ & $2(10,52 \%)$ & $1(20 \%)$ & $0 \quad(0 \%)$ & $3(8,10 \%)$ \\
\hline Pelícano & $6(66,67 \%)$ & $0 \quad(0 \%)$ & $0 \quad(0 \%)$ & $(0 \%)$ & $6(16,21 \%)$ \\
\hline Piquero & $0(0 \%)$ & $(0 \%)$ & $0 \quad(0 \%)$ & $(0 \%)$ & $0 \quad(0 \%)$ \\
\hline
\end{tabular}

Tabla 4. Abundancia y valores porcentuales de la garrapata Ornithodoros amblus asociada a diferentes intensidades de guano

\begin{tabular}{|c|c|c|c|c|c|}
\hline $\begin{array}{l}\text { Intensidades } \\
\text { de guano }\end{array}$ & Larva & Ninfa & hembra & Macho & Total \\
\hline Abundante & $4(33,33 \%)$ & $14(41,17 \%)$ & $7(53,84 \%)$ & $6(54,54 \%)$ & $31(44,28 \%)$ \\
\hline Medio & $2(16,67 \%)$ & $02(05,88 \%)$ & $2(15,38 \%)$ & $1 \quad(9,09 \%)$ & $07 \quad(10 \%)$ \\
\hline Pobre & $6 \quad(50 \%)$ & $14(41,17 \%)$ & $4(30,76 \%)$ & $4(36,37 \%)$ & $(40 \%)$ \\
\hline Ausente & $0 \quad(0 \%)$ & $04(11,76 \%)$ & $0 \quad(0 \%)$ & $0(0 \%)$ & $(05,7 \%)$ \\
\hline
\end{tabular}


Tabla 5. Abundancia valores porcentuales de la garrapata Ornithodoros amblus asociada a diferentes intensidades de viento

\begin{tabular}{llllrl}
\hline $\begin{array}{l}\text { Intensidades } \\
\text { de viento }\end{array}$ & Larva & Ninfa & Hembra & Macho & Total \\
\hline Fuerte & $3(25 \%)$ & $19(55,88 \%)$ & $9(69,23 \%)$ & $10(90,90 \%)$ & $41(58,57 \%)$ \\
Medio & $9(75 \%)$ & $12(35,29 \%)$ & $3(23,08 \%)$ & $01(9,10 \%)$ & $25(35,71 \%)$ \\
Ausente & $0(0 \%)$ & $03(08,83 \%)$ & $1(07,69 \%)$ & $0(0 \%)$ & $04(05,72 \%)$ \\
\hline
\end{tabular}

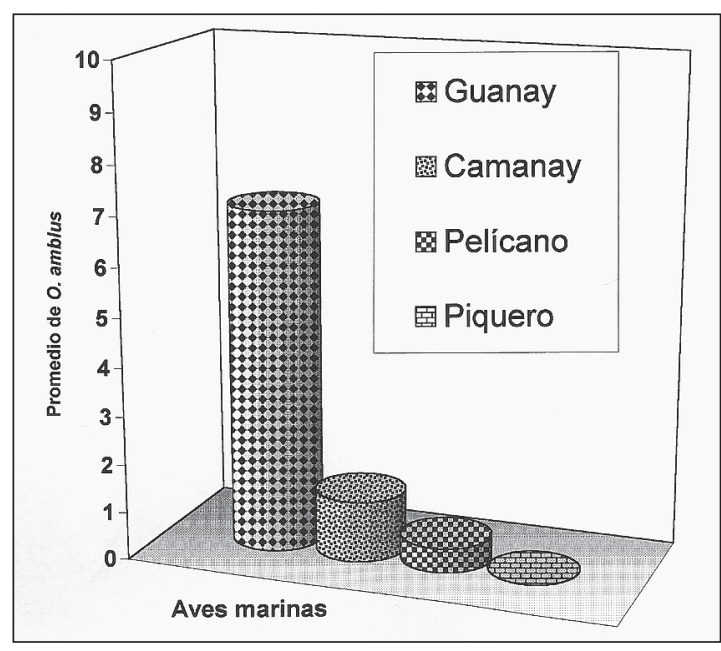

Figura 1. Relación entre la garrapatas $O$. amblus y las especies de aves marinas.

embargo, las categorías guano abundante y pobre en conjunto representaron el $84,28 \%$ del total de garrapatas censadas (Tabla 4). Se observó preferencia de las poblaciones de $O$. amblus con relación a la intensidad de viento, siendo el orden decreciente mayormente para todos los estadios de desarrollo, fuerte $>$ medio $>$ ausente $\left(\chi^{2}=\right.$ 14,49; g. 1. =6; P= 0,02). (Tabla 5).

\section{DISCUSIÓN}

La garrapata $O$. amblus tiene una distribución que se restringe principalmente al Perú ${ }^{5}$. Otra especie peruana de este género en aves marinas es $O$. spheniscus, parásito del pingüino de Humboldt Spheniscus humboldti ${ }^{14}$. El hallazgo de un ejemplar durante el estudio se explica por la existencia de una pequeña y reducida colonia de pingüinos en la Isla Mazorca, aunque fue hallada en una zona alejada al área muestreada.

Con relación al aspecto de la metodología de muestreo no existen resultados comparables a los obtenidos en el presente estudio. Duffy ${ }^{6}$ empleó cucharones de $500 \mathrm{~cm}^{3}$ para la colecta de guano, obteniendo 9,9 $\pm 0,7$ garrapatas (ninfas y adultos) en guanayes en épocas reproductivas. En el presente estudio se colectaron las garrapatas empleando una tela de tocuyo negro.

El número promedio de garrapatas por punto de muestro es aparentemente bajo $(3 \pm 3,6$ por $0,35 \mathrm{~m}^{2}$ ), quizás como consecuencia del ENSO 1997-1998 ${ }^{15}$. Lo que se explicaría debido a la escasez de alimento (disminución de aves marinas hospederas) que mermarían la población de estos ácaros ${ }^{6}$, Aunque las especies de garrapatas disminuyen como consecuencia del $\mathrm{ENSO}^{6}$, se recuperan aparentemente más rápidamente que las poblaciones de aves después de este fenómeno, debido a que el género Ornithodoros tiene la capacidad de sobrevivir por largos períodos sin alimentarse y a los pocos depredadores de esta garrapata $^{3,5}$.

Se hace evidente la relación entre la especie de ave guanera y el número de parásitos que se hallaron en el área que ocupaban (Figura 1). En el caso del guanay que presentó las mayores densidades de aves (15 aves $\mathrm{m}^{-2}$ ), presentó las mayores cantidades de garrapatas (Tabla 3). Duffy $^{6}$ halló que la distancia a las colonias de aves tenían relación con la abundancia de garrapatas, lo que se haría más evidente aún en el periodo reproductivo de las aves guaneras y determinaría la deserción de estas últimas. En contraste en el presente estudio se encontró que la abundancia de $O$. amblus no se encuentra relacionada con la presencia de aves. Sólo las larvas prefirieron localizarse en áreas asociadas a colonias de aves. Esto último podría explicarse, debido a que el estado larval de $O$. amblus requiere alimentarse de aves durante 4 a 6 d sobre el hospedero, en contraste con los otros estados de desarrollo como el ninfal y el adulto de 10 a $60 \mathrm{~min}^{5,8}$. Otros autores han encontrado una mayor población de ninfas de Ixodes scapularis Say en suelos de áreas 
naturales en Norteamérica en presencia de su hospedero, el venado de cola blanca Odocoielus virginianus ${ }^{16}$ y de Ixodes uriae en los pingüinos Aptenodytes patagonicus halli y Eudyptes chrysolophus en condiciones subantárticas ${ }^{17}$.

La relación del parásito con otro factor como la intensidad de viento (Tabla 5), haría necesaria la medición más cuantitativa y analítica de la fuerza de este, ya que zonas con viento de intensidad fuerte son las preferidas por las aves guaneras ${ }^{18}$. Además el viento facilita la dispersión de las garrapatas 5 .

\section{RESUMEN}

El objetivo de la presente investigación fue censar a la garrapata Ornithodoros amblus en la Isla Mazorca, Lima, Perú y su relación con la presencia de aves marinas, intensidad de sustrato y viento. El muestreo de las garrapatas se realizó los días 28 y 29 de febrero del año 1998. En cada punto de muestreo se colocó por espacio de tres minutos una tela de tocuyo negra de 0,35 $\mathrm{m}^{2}$. O. amblus presentó una distribución espacial amontonada. La abundancia de garrapatas no estuvo relacionada con la presencia de colonias de aves. Sólo las larvas de $O$. amblus estuvieron relacionadas con las colonias de aves. La preferencia de los diferentes estados de desarrollo de $O$. amblus siguió la siguiente secuencia: Guanay > Pelícano > Camanay. Los diferentes estados de desarrollo no se encontraron en relación con las intensidades de guano. Se observó preferencia de las poblaciones de $O$. amblus con relación a fuertes intensidades de viento. Se discute el efecto del Niño 1997-1998 sobre esta especie de garrapata.

\section{REFERENCIAS}

1.- ESTRADA A P, JONGEJAN F. Ticks feeding on humans: a review of records on human-biting Ixodoidea with special reference to pathogen transmission. Exp Appl Acarol 1999; 23: 685-715.

2.- EVANS D E, MARTINS J R, GUGLIELMONE A A. A Review of the ticks (Acarina: Ixodida) of Brazil, their hosts and geographic distribution- 1 . The state of Rio Grande do Sul, southern Brazil. Mem Inst Oswaldo Cruz 2000; 95: 453-70.

3.- HELMY N. Seasonal abundance of Ornithodoros $(O$.) savignyi and prevalence of infection with Borrelia spirochetes in Egypt. J. Egypt Soc. Parasitol 2000; 30: 607-719.

4.- KEIRANS J E, HUTCHENSON H J, OLIVER J H Jr. Ornithodoros (Alectorobius) capensis, a parasite of seabirds, established along the southeastern seacost of the United States. J Med Entomol 1992; 29: 371-3.

5.- IANNACONE J, DALE W. Ornithodoros (Alectorobius) amblus (Acarina: Ixodoidea: Argasidae): garrapata parásita del guanay en el Perú. Rev Per Ent 1997; 40: 21-6.

6.- DUFFY D C. The ecology of parasitism on densely nesting Peruvian seabird. Ecology 1983; 64: 110-9.

7.- NEED J T, DALE W E, KEIRANS J E, DASCH G A. Annotated List of Ticks (Acari: Ixodidae, Argasidae) reported in Peru: distribution. Host and bibliography. $\mathbf{J}$ Med Entomol 1991; 28: 590-7.

8.- SCHUMAKER T T, MORI C M, FERREIRA C S. Experimental infestation of Gallus gallus with Ornithodoros (Alectorobius) amblus (Ixodoidea: Argasidae). J Med Entomol 1997; 34: 521-6.

9.- DUFFY D C, MERLEN G. Seabird densities and aggregations during the 1983 El Niño in the Galapagos Island. Wilson Bull 1986; 98: 588-91.

10.- MANS B J, LOUW A I, NEITZ A W. Evolution of hematophagy in ticks: common origins from blood coagulation and platelet aggregation inhibitors from soft ticks of the genus Ornithodoros. Mol Biol Evol 2002; 19: 1695-705.

11.- IANNACONE J A. Olfersia fossulata Macquart (Diptera : Hippoboscidae) ectoparásita de las aves guaneras. Rev Per Ent 1996; 39: 119-20.

12.- BAETY B, MARQUART W. The Biology of disease vectors. University Press of Colorado. USA. 1996.

13.- KHALIL G M, HOOGSTRAAL H. The life cycle of Ornithodoros (Alectorobius) amblus (Acari: Ixodoidea: Argasidae) in the laboratory. J Med Entomol 1981; 18: $134-9$

14.- HOOGSTRAAL H, WASSEF H Y, HAYS C, KERIANS J E. Ornithodoros (Alectorobius) spheniscus n. sp. (Acarina: Ixodoidea: Argasidae Ornithodoros (Alectorobius) capensis group), a tick parasite of the Humboldt penguin in Peru. J Parasitol 1985; 71: 63544.

15.- IANNACONE J A, ALVARIÑO L, MORENO R et al. Culícidos (Diptera) del río Chillón y sectores adyacentes de la Provincia constitucional del Callao, Perú, durante el Niño 1997-1998. Acta Ent Chile 2000; 24: 51-60.

16.- DUFFY D C, CAMPBELL S R, CLARK D et al. Ixodes scapularis (Acari: Ixodidae) deer tick mesoscale populations in natural areas: effects of deer, area, and locations. J Med Entmol 1994; 31: 152-8.

17.- FRENOT Y, OLIVEIRA E, GAUTHIER M, DEUNFF $\mathrm{J}$ et al. Life cycle of the tick Ixodes uriae in penguin colonies: relationships with host breeding activity. Int J Parasitol 2001; 31:1040-7.

18.- VOGT W Q. Aves guaneras. Bol Comp Ad Guano 1942; 17: $1-132$. 